\title{
Calcium complexation by organic matter in calcareous sediments
}

\author{
S.E. BONE', J. CliFF'r, K. WeAVER', C.J. TAKACS ${ }^{\prime}$, S. \\ ROYCROFT $^{3}$, S. FENDORF ${ }^{3}$, J.R. BARGAR'
}

'SLAC National Accelerator Laboratory, USA,

*correspondance: shbone@slac.stanford.edu

2Environmental Molecular Sciences Laboratory, USA

${ }^{3}$ Stanford University, USA

Soils and sediments host large stores of organic carbon which can be released to the atmosphere upon mineralization. $\mathrm{Ca}$ has the potential to play a key role in preventing mineralization of this organic carbon by promoting mineral protection. It has long been recognized that $\mathrm{Ca}$ forms cation bridges that link together negatively charged functional groups from organics and mineral surfaces, stabilizing organic carbon via mineral sorption. However, there is little direct experimental observation of the coordination between $\mathrm{Ca}$, organic matter (OM), and minerals in sediments, which can be used to determine the molecular-scale mechanism by which $\mathrm{Ca}$ bridges $\mathrm{OM}$ to mineral surfaces.

In the current work, we examined whether and how $\mathrm{Ca}$ was associated with $\mathrm{OM}$ in calcareous sediments. We sought to determine whether Ca was co-associated with OM and specific minerals, such as iron oxides or clays; as well as what types of OM Ca was associated with. Additionally, we sought to determine the local coordination environment of $\mathrm{Ca}$ complexed by $\mathrm{OM}$ to assess whether it participated in briding interactions.

To this end, sediments were subjected to density fractionation to separate organic and mineral components of the sediments. This technique yieded a particulate organic matter fraction, a fraction containing organic-mineral aggregates, and a fraction that was mostly mineral, with a small amount of organics. In each fraction, the colocalization of $\mathrm{Ca}$ with different organic species and with $\mathrm{Al}$, Si and Fe minerals was probed using nano secondary ion mass spectrometry (NanoSIMS) and scanning transmission X-ray microscopy (STXM) at the scale of ca. $100 \mathrm{~nm}$. Ca speciation was probed further using (micro-)X-ray absorption near edge strcuture (XANES) and extended Xray absorption fine structure (EXAFS) spectroscopy. Our analysis sheds new light on the role that $\mathrm{Ca}$ plays in stabilizing OM in calcareous sediments, thereby limiting its mineralization and release as $\mathrm{CO}_{2}$ into the atmosphere. 